\title{
Research on Spread Spectrum Communication Technique based on Binary Offset Subcarrier Frequency Sweep and Related Methods
}

\author{
Wenzhun Huang ${ }^{1, a^{*}}$, Yuting Zhang ${ }^{1, b}$, Xinxin Xie ${ }^{1, c}$ and Shanwen Zhang ${ }^{1, d}$ \\ ${ }^{1}$ Department of Electronic Information Engineering, Xijing University, Xi'an 710123, China \\ No. 1, Xijing Road, Chang An District, Xi'an, Shaanxi Province, China

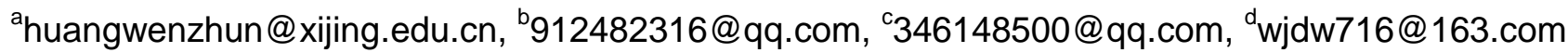

Keywords: Spread Spectrum Communication; Binary Offset; Frequency Sweep; Asymptotic Bounds; Fast Fourier Transform (FFT); Multicarrier Modulation.

\begin{abstract}
In this paper, we conduct theoretical research and numerical simulation on the spread spectrum communication technique based on binary offset subcarrier frequency sweep and related methods. At present, most of the spread spectrum signal generation did not give the effect of channel filter and a large number of related studies have shown that channel non-ideal properties of channel filter will produce great influence to the precision. Therefore, it is best to consider when the signal will generate channel filter effects on signal. The maximum differential values between different paths which is also known as multipath time delays are studied in this research. Multipath propagation phenomenon usually digital communication element in the overlap problem and causes the judgment of errors and error occurs which affect the improvement of wireless communication speed and reliability of communication. Therefore, we conduct modification based on the related prior research result. In our method, more specific models are analyzed with inductions and the experimental simulation proves the effectiveness of the proposed approach. In the future, we have scheduled to do more in-depth analysis to optimize the current approach.
\end{abstract}

\section{Introduction}

The traditional approach of designing the communications system could be dated back to the Shannon's time which was to treat coding and modulation as separate procedures that can each be studied and optimized individually. However, from the perspective of signal detection, more natural think indicated that they are a single and unified process. As for the principle for the first time using trellis coded modulation method, short wave transmission way pf the main has two kinds: one for the ground wave, another for sky wave. The ground wave propagated along the surface of the earth, through this way the propagation distance is mainly decided by the surface dielectric properties. It is enhanced by the ground wave attenuation with the increase of frequency that short wave propagation in ground wave manner will use the commonly used transmission power while short wave propagation distance up to only a few hundred kilometers. Therefore, the ground is not the main approach to spread use in shortwave communication. The generation of spread spectrum signal generally could be separated into two ways [1]. After one is using relevant models, this model can effectively shorten the simulation time, but the results of related after modeling will ignore the code level of detail. Before one is related to model, this model can achieve high fidelity of spread spectrum signal is generated and the disadvantage is that the simulation computation which results that the efficiency is not high. At present, most of the spread spectrum signal generation did not give the effect of channel filter and a large number of studies have shown that channel non-ideal properties of channel filter will produce great influence to the precision, so it is best to consider when to signal generated channel filter effects on signal. 
To realize the spread spectrum signal blind algorithm, we first of all need to implement effective for signal detection and signal parameter estimation in order to solve this problem and the scholars put forward the square frequency doubling method, time domain autocorrelation method and related wavelet transform and higher-order method such as a variety of methods. However, these methods have certain priori information or requirements. In addition, these algorithms suffer from the low SNR fall effect [2-5].

To deal with the mentioned drawbacks and disadvantages, we conduct research on spread spectrum communication technique based on binary offset subcarrier frequency seep and related methods. With microelectronics technology, computer technology and the continuous development of digital signal processing technology, short-wave radio communication technology is well adopted in adaptive transceiver and adaptive modems which makes a breakthrough and made it possible to short-wave wireless communication technology to solve the high noise level, fading and multipath propagation channel time-varying characteristics in the direction of the digital and low bit error rate as well as the high rate of continued development. To specifically illustrate the system, in this paper FFT algorithms proposed in the literature for cognitive radio applications are investigated which will be discussed below [6-10].

\section{The Proposed Spread Spectrum Communication Technique}

The OFDM Based Multicarrier Environment. OFDM reduces the time dispersion effect of multipath channels encountered with high data rates by using cyclic prefix. For the system, the signal model of baseband signal processing before available is represented in the Figure 1. Here independent set signal filter and noise filter which makes the model is more general.

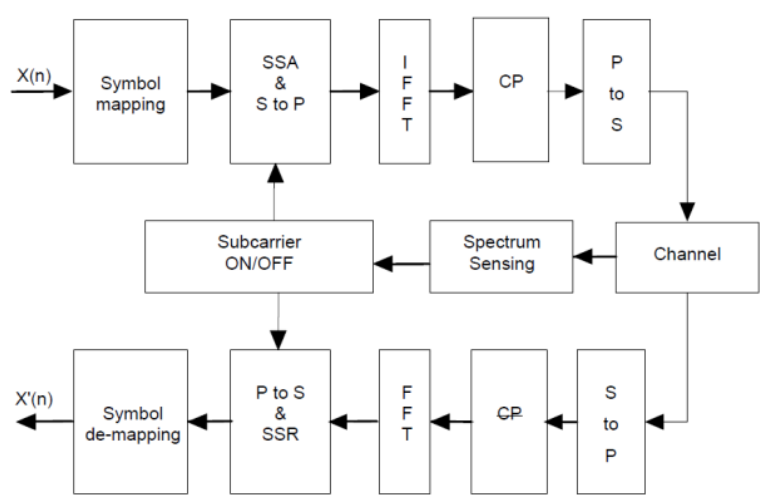

Fig. 1 The Selective Subcarrier Allocation Pattern for the System

Electromagnetic wave propagation through the different paths and could eventually reach the terminal receiver phenomenon which is known as multipath propagation. Due to the propagation path length is different, each arrive the electromagnetic wave signal receiver is different. Multipath propagation phenomenon is usually related to digital communication element in the overlap problem, which causes the judgment of errors and error occurs which affected the improvement of wireless communication speed and reliability of communication. Because of shortwave radio communication equipment antenna beam wide, ray divergent is larger and because the earth's ionosphere is layered, so in a short wave radio signal propagation of electromagnetic waves exist in different propagation path which is the multipath propagation phenomenon. Short-wave multipath propagation in wireless communications will generate two problems: one is decline, the other one is time delay. Shortwave electromagnetic signal travels in the earth's ionosphere due to the randomness of the ionosphere electrical characteristics change and it will cause different signal transmission path and the energy absorption of random changes which makes the receiver the level irregular change. Signal amplitude presents random changes and this problem is called decline. Decline problems had a great influence on the reliability and stability of the short-wave radio communication, sometimes even lead to 
communication breakdown. Multipath propagation also makes the transmission signal distortion or limited transmission channel bandwidth.

Implementation and System Modelling. Spread spectrum signal spectrum correlation function is a basic model. The formula one shows the sample received signal.

$$
x(t)=s(t)+n(t)=d(t) p(t) \cos \left(\left(2 \pi f_{0} t+\psi\right)+\psi_{0}\right)+n(t)
$$

Spread spectrum signal is a random signal characteristic of cycle stationary signal which belongs to the cycle stationary signal. Eliminate the loss of orthogonal spreading code set any transmitted OFDM system and maintain the same data rate of the traditional OFDM. In a scheme, the multicarrier system is divided into multiple subsystems and orthogonal WH variable length coding for each subsystem maintenance data rate which is shown as the expression 2.

$$
R_{x}^{a}(\tau)=\lim _{T \rightarrow \infty} \frac{1}{T} \int_{-T / 2}^{T / 2} R(t+\tau / 2, t-\tau / 2) e^{-j 2 \pi t} d t
$$

In a signal transmission interval, the source sends the information to occupy one or more of the available frequency gap in a similar manner of pseudo random code that $\mathrm{FH}$ sequences can jump in wide frequency band. Baseband signal is the preliminary modulation before entering the carrier modulation. The spectrum respectively upward move down and we can therefore get the time average of the two type of cross-correlation shown as the equation 3.

$$
S_{X_{T}}^{a}(f)=\frac{1}{\Delta t} \int_{-\Delta t / 2}^{\Delta t / 2} X_{T}(s, f+a / 2) X_{T}^{*}(s, f-a / 2) d s
$$

At the receiver side, the system first recover RF signal from the transmitter to send the FH synchronization signal, the frequency hopping sequence control at the receiving end of the changes of frequency and frequency hopping signal synchronization. Through using the local oscillator signal demodulation receiving frequency, we could hope the signal and then obtain baseband signal. Frequency hopping technology is a kind of favorable Rayleigh fading resistance technology which can reduce the depth of the mixed and related time delay. In multiple accesses communication system which can improve the spectrum efficiency, strong anti-interference, fading resistance, resistance to intercept and monitor resistance, flexible spectrum use does not require the use of a continuous spectrum which makes it much easier to frequency planning. The following formulas show the corresponding features.

$$
\left|S_{X_{T}}^{a}(f=0)\right|=\left\{\begin{array}{cc}
\frac{1}{2 T_{c}}\left|Q\left(f_{0}+a / 2\right) Q^{*}\left(f_{0}-a / 2\right)\right| & a=k / T \\
\frac{1}{4 T_{c}}\left|Q\left(f_{0}+a / 2\right)\right|^{2} & a=\mp 2 f_{0}+k / T_{c}
\end{array}\right.
$$

When the electromagnetic wave frequency transmission using reaches system can withstand the highest frequency, multipath time delay will be reduced to the minimum value of response and transmission will spread with the ordinary single jump very close. If the work frequency of the system could be reduced, at the same time also can cause the rise of multipath time delay value. In the Figure 2, we illustrate the revised structure of the initial system. 


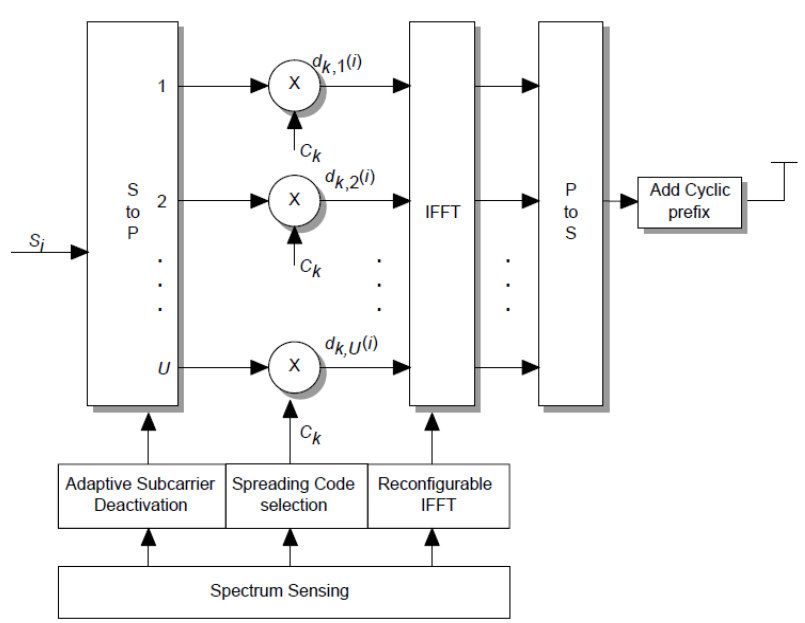

Fig. 2 The Revise Structure for the Proposed System

Contemporary use of the modem in shortwave radio communications equipment mainly include the serial and parallel two architectural approaches including serial mode using single carrier modulation to realize signal to send and has a high requirement on the equilibrium and parallelism is the parallel points will need to send information from several different sub-carrier generally in parallel. Each subcarrier frequency will be different, but the group adopts the relevant filter in the receiver to select each channel so the requirements for each child channel should have a certain frequency range used for convenient between filtering operation. Spread spectrum signal amplitude spectrum correlation function is not zero but in the actual cases the noise exist which can choose the appropriate threshold to compare with amplitude to estimate the early phase. Therefore, referring to the state-of-the-art approaches we set the value in the formula 5.

$$
\gamma=\sqrt{2 \sigma_{s}^{2} \operatorname{Ln}\left(\frac{1}{P_{f}}\right)}
$$

The main way of spread spectrum communication mainly include direct sequence spread spectrum, frequency hopping and jumping and so on. In shortwave radio, communications are mainly composed of frequency hopping and also devices use a small amount of direct sequence spread spectrum, frequency hopping or combined technology of frequency hopping and jumping. The corresponding result for the system is shown in the next section.

\section{Experiment Result and Simulation}

The author through the Monte Carlo simulation experiment for listed on signal spectrum correlation method is used to estimate the carrier frequency, symbol rate and initial phase and we adopt the experimental repetitions for 500 times. In the Figure 3, we show the result. Visible with the decrease of the signal-to-noise ratio, the estimate error increases gradually but increase acquisition time can improve the estimation precision. Therefore, under the condition of existing increase or increase the length of the data acquisition time can be better estimates of the effect which is worth further study. In the Figure 4, we show the result for bit error rate of different systems. The result indicate that without a pseudo random code sequence and related parameters under the condition of a priori information, the method can complete low SNR of signal detection and carrier frequency and code rate, in the early phase of estimate and because the estimation algorithm is on non-zero cyclic frequency cyclic spectrum of one-dimensional search for many times, it will effectively improve the computation efficiency. Visible with the decrease of the signal-to-noise ratio, the estimate error will increase gradually, but increase acquisition time can improve the estimation precision. Therefore, under the condition of existing increase or increase the length of the data acquisition time can be better estimates of the effect which is worthy of further study. 


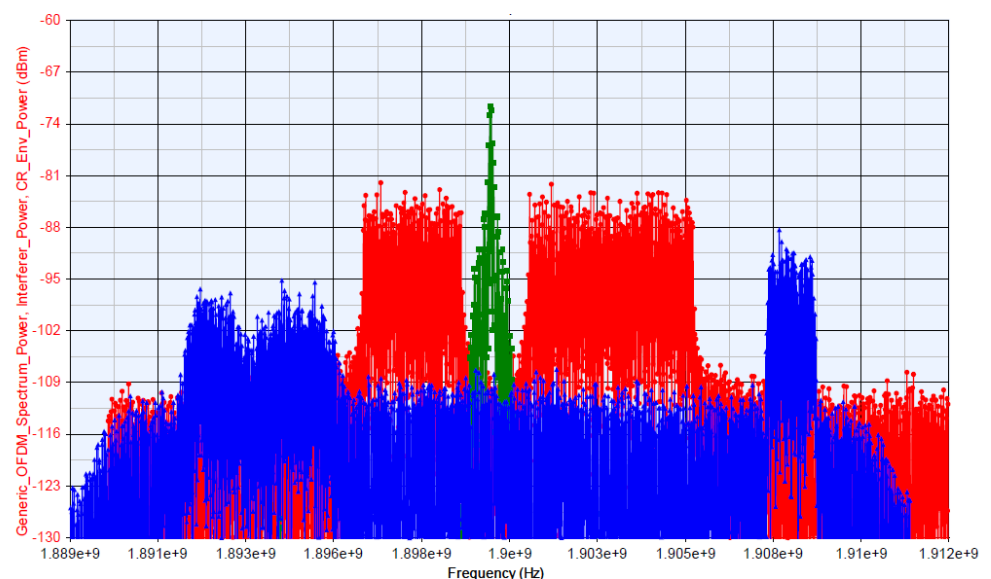

Fig. 3 The Experimental Result for the Proposed Methodology

\section{Conclusion and Summary}

In this paper, we conduct the novel research on spread spectrum communication technique based on binary offset subcarrier frequency sweep and related methods. The ground wave propagated along with the surface of the earth is researched in our methodology, through this way the propagation distance is mainly decided by the surface dielectric properties. It is enhanced by the ground wave attenuation with the increase of frequency and the proposed model deal with the drawbacks properly. The experimental result reflects the fact that our approach outperforms other methodologies and obtains better accuracy. In the future, we plan to optimize the current method to obtain better result.

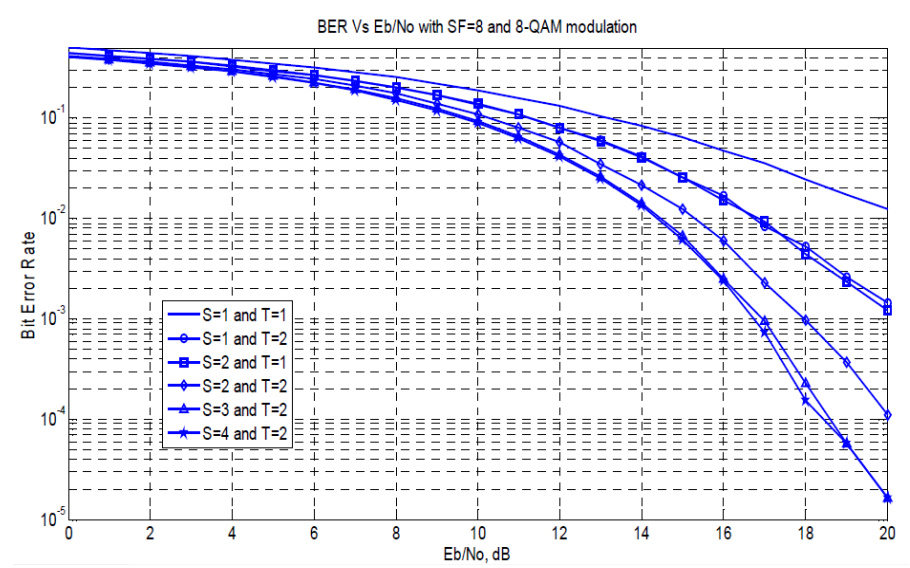

Fig. 4 The Bit Error Rate of Different Systems

\section{Acknowledgement}

This work is supported by Shaanxi Provincial Education Department (Program No. 14JK2156).

\section{References}

[1] Landolsi M A. Signal Design for Improved Multiple Access Capacity in DS-UWB Communication[J]. Wireless Personal Communications, 2015, 80(1):1-15.

[2] Thombre S, Nurmi J. Software Simulators and Multi-Frequency Test Scenarios for GALILEO[J]. Signals \& Communication Technology, 2015:289-321.

[3] Ito T, Okamoto A. Coherent Parallel Copying of Holograms Recorded by Spatial Spread-Spectrum Multiplexing[J]. Japanese Journal of Applied Physics, 3816, 46:3816-3821. 
[4] Roufoogaran, R., Li, Q., \& Behzad, A. (2007). Method and System for a Compact and Power Efficient Local Oscillator Generation Architecture. US, US20080139115 A1.

[5] Merritt S. Thermal signature identification system (TheSIS): a spread spectrum temperature cycling method[J]. Photonic Instrumentation Engineering II, 2015.

[6] Masucci, A. M., Belmega, E. V., \& Fijalkow, I. (2014). Optimal blockwise subcarrier allocation policies in single-carrier fdma uplink systems. Eurasip Journal on Advances in Signal Processing.

[7] Vinoth Babu, K., \& Ramachandra Reddy\& L. Balakrishnan, G. (2014). Spectral efficiency improvement in ofdma systems with modified adaptive subcarrier spacing based resource allocation algorithm. Hkie Transactions, 21, 3, 158-169.

[8] Wang, Y., \& Chen, W. (2014). A kernel-based ici self-cancellation scheme using constrained subcarrier combiners. Signal Processing, 101, 8, 142-150..

[9] D. F. Z., \& Wang, Y. F. (2014). A fast and high efficiency carrier offset estimator for ofdm. Applied Science Materials Science \& Information Technologies in Industry.

[10]Dong-hua, C. (2014). Performance analysis of cooperative ofdm systems in presence of carrier frequency offset and in high mobility environment. Journal of Huaqiao University. 